\title{
GENERATION OF GENERATORS OF HOLOMORPHIC SEMIGROUPS
}

\section{CHRISTIAN BERG, KHRISTO BOYADZHIEV and RALPH DELAUBENFELS}

(Received 26 November 1990)

Communicated by P. G. Dodds

\begin{abstract}
We construct a functional calculus, $g \mapsto g(A)$, for functions, $g$, that are the sum of a Stieltjes function and a nonnegative operator monotone function, and unbounded linear operators, $A$, whose resolvent set contains $(-\infty, 0)$, with $\left\{\left\|r(r+A)^{-1}\right\| \mid r>0\right\}$ bounded. For such functions $g$, we show that $-g(A)$ generates a bounded holomorphic strongly continuous semigroup of angle $\theta$, whenever $-A$ does.

We show that, for any Bernstein function $f,-f(A)$ generates a bounded holomorphic strongly continuous semigroup of angle $\pi / 2$, whenever $-A$ does.

We also prove some new results about the Bochner-Phillips functional calculus. We discuss the relationship between fractional powers and our construction.
\end{abstract}

1991 Mathematics subject classification (Amer. Math. Soc.): 47 A 60, 47 B 44, 47 D 05.

\section{Introduction}

It is natural to ask what properties of an operator, $A$, are inherited by its resolvents, $(t+A)^{-1}$. More generally, one may consider Stieltjes functions,

$$
g(z) \equiv a+\int_{0}^{\infty} \frac{1}{z+t} d \lambda(t),
$$

where $a \geq 0$ and $\lambda$ is nondecreasing. The class of Stieltjes functions also includes fractional powers $z \mapsto z^{\alpha},-1 \leq \alpha<0$.

(C) 1993 Australian Mathematical Society 0263-6115/93 \$A2.00+0.00 
In this paper, we show that, if the image of $A$ is dense, and $-A$ generates a bounded holomorphic semigroup of angle $\theta$, then the same is true of $g(A)$, defined to be the closure of

$$
g(A) x \equiv a x+\int_{0}^{\infty}(t+A)^{-1} x d \lambda(t), x \in \text { image of } A,
$$

where $g$ is as in (1.1). (See Section 5.)

More generally, suppose $\left\{e^{-t A}\right\}_{t \geq 0}$ is a strongly continuous semigroup of operators generated by $-A$. From the beginning of the theory of such semigroups, certain functional calculi

$$
f \mapsto f(A)
$$

have been developed for appropriate classes of functions, $f$, defined on the spectrum of $A$. For such a functional calculus, a fundamental problem is for what functions, $f$, the operator $-f(A)$ is again a generator, that is, $\left\{e^{-t f(A)}\right\}_{t \geq 0}$ is a strongly continuous semigroup of operators.

A functional calculus construction that has received much attention is one constructed by Bochner and Phillips ([8, 38]), for the Bernstein functions (see Section 2). This functional calculus has the property that $-f(A)$ generates a strongly continuous contraction semigroup whenever $-A$ does. A natural and important question was asked by Kishimoto and Robinson in [28, page 63]: if $-A$ generates a bounded strongly continuous holomorphic semigroup, is the same true for $-f(A)$, where $f$ is a Bernstein function?

In this paper, we give two partial answers to this open question, that cover the cases of most interest. We show that, when $-A$ generates a bounded strongly continuous holomorphic semigroup of angle $\theta$, then the same is true of $-f(A)$ when $f$ is a nonnegative operator monotone function (see Section 5). This class of functions is contained in the class of Bernstein functions and includes such functions as fractional powers $z \mapsto z^{\alpha}(0<\alpha<1), z \mapsto \log (1+z)$, $z \mapsto \sqrt{z} \arctan (1 / \sqrt{z})$. When $A$ has dense range, the same is true for sums of Stieltjes functions and nonnegative operator monotone functions (Section 6); this includes functions not in the Bernstein class.

Our results in this regard are actually sharper than an affirmative answer to the original question in [28], since we show that the angle of the semigroup is preserved.

When the angle of the holomorphic semigroup generated by $-A$ equals $\pi / 2$, then we show that the answer to the open question above is affirmative, for any Bernstein function $f$. More than that, we show that this angle is preserved, that is, $-f(A)$ generates a bounded holomorphic strongly continuous semigroup of 
angle $\pi / 2$ whenever $-A$ does. This result may be applied to $A \equiv-\triangle$, where $\triangle$ is the Laplacian, on $L^{p}\left(\mathbb{R}^{n}\right), 1 \leq p<\infty$,or $C_{0}\left(\mathbb{R}^{n}\right)$, or more general elliptic operators (Section 7).

The desired property here that we are trying to preserve is generating a bounded holomorphic strongly continuous semigroup. In order that $-A$ generate a bounded holomorphic strongly continuous semigroup of angle $\theta$, it is necessary, but not sufficient, that the spectrum of $A$ be contained in $\overline{S_{\pi / 2-\theta}}$, where $S_{\psi} \equiv\left\{r e^{i \phi}|| \phi \mid<\psi, r>0\right\}$. If we define an operator, $A$, to be of type $\theta$ (see $[34,41,20,48]$ ) whenever $-A$ generates a bounded holomorphic strongly continuous semigroup of angle $(\pi / 2-\theta)$, then our results may be interpreted as a type mapping theorem (see Corollary 6.3), which is a spectral mapping theorem and more:

$$
\text { type of } f(A)=\theta \text {, when } f: S_{\psi} \rightarrow S_{\theta} \text {, and } A \text { is of type } \psi,
$$

for all sums of Stieltjes functions and nonnegative operator monotone functions $f$.

It is interesting that not all holomorphic functions that map the right half-plane to itself preserve type, even on a Hilbert space; this is a consequence of the results in [34]. The most that can be said is that, if $-A$ generates a bounded holomorphic strongly continuous semigroup, then $-f(A)$ generates a $C$-semigroup (see [14] for the definition and basic properties), with $C \equiv(1+A)^{-1}$ (see [10]), or $(1+A)^{-r}, \forall r>0$ (see [15]), when $f$ is a holomorphic function that maps the right half-plane to itself.

Thus it is natural to ask what additional conditions on $f$ are necessary to guarantee that $f$ preserves type. In Section 6, we show that it is sufficient that $f$ be a sum of a Stieltjes function and a nonnegative operator monotone function. Alternatively, one could ask what additional conditions on $A$ will guarantee that $-f(A)$ is of type $\theta<\pi / 2$, whenever $f$ is a holomorphic function that preserves the right half-plane; see [34,41] and [20], for some constructions ( $H^{\infty}$ functional calculi) that produce operators, $A$, that will have this property. The choice here is between having a larger class of functions or a larger class of operators. In this paper, we chose the latter; our results are valid for any operator, $A$, such that $-A$ generates a bounded, strongly continuous holomorphic semigroup.

This paper also contains some new results about fractional powers and their relationships with our functional calculus and the Bochner-Phillips functional calculus, that may be of some independent interest. This includes results about the behaviour of the functional calculus under compositions.

Sections 2 and 3 contain some preliminary results, about Bernstein functions 
and the Bochner-Phillips functional calculus (Section 2) and bounded holomorphic strongly continuous semigroups (Section 3). Section 4 presents some new results about fractional powers and the Bochner-Phillips functional calculus. In Section 5, we introduce, for operators of type $\theta<\pi$ (this includes, but is not limited to, operators, $A$, such that $-A$ generates a strongly continuous bounded semigroup), a functional calculus construction for Stieltjes functions and nonnegative operator monotone functions. In Section 6 we prove that type is preserved by the construction in Section 5 , that is, whenever $-A$ generates a bounded holmorphic strongly continuous semigroup of angle $\theta$, then the same is true of $-f(A)$, whenever $f$ is a sum of a Stieltjes function and a nonnegative operator monotone function. In Section 7, we show that, for any Bernstein function $f,-f(A)$ generates a bounded holmorphic strongly continuous semigroup of angle $\pi / 2$ whenever $-A$ does.

\section{Bernstein functions and the functional calculus of Bochner-Phillips}

A completely monotone function is a function $f \in C^{\infty}(0, \infty)$ satisfying

$$
(-1)^{n} f^{(n)}(x) \geq 0, \quad \forall x>0, \quad n=0,1,2, \ldots
$$

These functions, according to the famous Bernstein Theorem, are characterized by the representation

$$
f(x)=\int_{0}^{\infty} e^{-x t} d \sigma(t)
$$

where $\sigma$ is a nondecreasing function on $[0, \infty)([7,46,47])$. In order to define a functional calculus $f \mapsto f(A)$, when $-A$ generates a bounded strongly continuous semigroup, such that $-f(A)$ is again a generator, Bochner introduced a class of functions, $f$, for which $x \mapsto e^{-t f(x)}$ is completely monotone $\forall t \geq 0$ [8]. The following theorem is due to him.

THEOREM 2.1. Suppose $f \in C([0, \infty)) \cap C^{\infty}((0, \infty))$ is nonnegative. Then the following are equivalent.

(i) $g \circ f$ is completely monotone whenever $g$ is completely monotone.

(ii) $f^{\prime}$ is completely monotone.

(iii) $f$ has the representation

$$
f(x)=a x+b+\int_{0}^{\infty}\left(1-e^{-x t}\right) \frac{d \sigma(t)}{t},
$$


where $a, b \geq 0$ are constants and $\sigma$ is a nondecreasing function on $[0, \infty)$ such that $\int_{1}^{\infty} d \sigma(t) / t<\infty$.

(iv) $\exists$ a continuous convolution semigroup of nondecreasing functions $\left\{\sigma^{t}\right\}_{t \geq 0}$ such that

$$
e^{-t f(x)}=\int_{0}^{\infty} e^{-x s} d \sigma^{t}(s), \forall x, t \geq 0,
$$

and $\int_{0}^{\infty} d \sigma^{t}(s) \leq 1$. (For details, see $[8,9,7,38,28]$ ).

The functions $\sigma$ and $\sigma^{t}$ may be normalized in such a way as to make them uniquely determined by $f$.

Motivated by (i) above, Bochner called these functions "completely monotone maps". However, they are better known as Bernstein functions. We denote by $\mathscr{B}$ the class of Bernstein functions. $\mathscr{B}$ is obviously a convex cone. The representation (iv) leads to the functional calculus

$$
e^{-t f(A)}=\int_{0}^{\infty} e^{-s A} d \sigma^{t}(s),
$$

suggested by Bochner [8] and developed by Phillips [38]. The following theorem is one of the fundamental results in the theory of semigroups of operators.

THEOREM 2.2. (Bochner-Phillips) Suppose $\left\{e^{-t A}\right\}_{t \geq 0}$ is a contraction (positive) strongly continuous semigroup and $f \in \mathscr{B}$ is represented by (iii) of Theorem 2.1. Then

$$
x \mapsto a A x+b x+\int_{0}^{\infty}\left(x-e^{-t A} x\right) \frac{d \sigma(t)}{t}, x \in \mathscr{D}(A)
$$

is a closable operator, the closure of which is denoted $f(A)$, and $-f(A)$ generates a contraction (positive) strongly continuous semigroup

$$
e^{-t f(A)} x \equiv \int_{0}^{\infty} e^{-s A} x d \sigma^{i}(s)
$$

for $t \geq 0, x \in X$. If $a \neq 0$, then $\mathscr{D}(f(A))=\mathscr{D}(A)$ and (2.3) itself is closed.

Note that if $\left\|e^{-t A}\right\| \leq M, \forall t \geq 0$, then $\left\|e^{-t f(A)}\right\| \leq M \int_{0}^{\infty} d \sigma^{t}(s) \leq M, \forall t \geq$ 0 . The Bernstein functions naturally extend to the closed right half-plane $\overline{R H P}$, $R H P \equiv\{z \in \mathbf{C} \mid \operatorname{Re}(z)>0\}$, via the representation (iii) in Theorem 2.1. Also, they preserve $\overline{R H P}$, since $\operatorname{Re}\left(1-e^{-z t}\right) \geq 0$, when $\operatorname{Re}(z) \geq 0$. 
A natural and important question was asked by Kishimoto and Robinson, in $\left[28\right.$, p. 63]: If $\left\{e^{-t A}\right\}_{t \geq 0}$ is a bounded holomorphic strongly continuous semigroup, is the same true for $\left\{e^{-t f(A)}\right\}_{t \geq 0}$, when $f \in \mathscr{B}$ ? (Note that the Bernstein functions preserve sectors of the form $S_{\psi} \equiv\{z \in \mathbf{C}|| \arg (z) \mid<\psi\}$, for $|\psi|<\pi / 2$. This is a property of all holomorphic functions which preserve RHP and $(0, \infty)$.)

In the present paper we shall prove that the answer to that question is positive for a large class $\mathscr{R}$ of functions in $\mathscr{B}$. The class $\mathscr{R}$ is described in Section 4 .

There is one classical example of functions in $\mathscr{B}, f(z)=z^{\alpha}, 0<\alpha<1$, with the important property that, if $e^{-t A}$ is a bounded strongly continuous semigroup, then $\left\{e^{-t A^{\alpha}}\right\}$ is a bounded holomorphic strongly continuous semigroup ([49, 50]). The functions $z^{\alpha}$ are in the class $\mathscr{R}$ and we prove that every function in $\mathscr{R}$ that maps RHP into $S_{\psi}$, for some $\psi<\pi / 2$, has this property.

\section{Bounded holomorphic semigroups and operators of type $\Theta$}

DEFINITION 3.1. $S_{\psi} \equiv\left\{r e^{i \phi}|r>0,| \phi \mid<\psi\right\}$.

DEFINITION 3.2. Suppose $0<\psi \leq \pi / 2$. The strongly continuous semigroup $\left\{e^{-t A}\right\}_{t \geq 0}$ is a bounded holomorphic semigroup of angle $\psi$ if it extends to a holomorphic family of bounded operators $\left\{e^{-z A}\right\}_{z \in S_{\psi}}$ that is bounded and strongly continuous in $\overline{S_{\phi}}$, whenever $0<\phi<\psi$.

General information on bounded holomorphic semigroups, including Theorem 3.4 below, may be found in $[13,22,37]$.

DEFINITION 3.3. If $\psi<\pi$, the operator $A$ is said to be of type $(\psi, M)$ if $A$ is closed and densely defined, $\sigma(A) \subseteq \overline{S_{\psi}}$ and $\exists M<\infty$ such that

$$
\left\|z(z-A)^{-1}\right\| \leq M, \quad \forall z \notin \overline{S_{\psi}}
$$

(see [27]).

If $\theta<\pi$, we will say that $A$ is of type $\theta$ if $\forall \psi \in(\theta, \pi) \exists M_{\psi}<\infty$ such that $A$ is of type $\left(\psi, M_{\psi}\right)$. (See $\left.[41,34,48,44]\right)$

THEOREM 3.4. Suppose $0 \leq \theta<\pi / 2$. Then $A$ is of type $\theta$ if and only if $-A$ generates a bounded holomorphic semigroup of angle $\pi / 2-\theta$. 
THEOREM 3.5. ([16]) Suppose $A$ is an operator of type $\theta, n \in \mathbb{N}$, and $n \theta<$ $\pi / 2$. Then $A^{n}$ is of type $n \theta$.

COROLLARY 3.6. Suppose $A$ is an operator of type $\theta, n \in \mathbb{N}$, and $n \theta<\pi$. Then $A^{n}$ is of type $n \theta$.

Proof. All that needs to be shown is that $A^{2}$ is of type $2 \theta$, whenever $A$ is of type $\theta<\pi / 2$. Fix $\psi>2 \theta$. $\exists M_{\psi}$ such that $\left\|z(z-A)^{-1}\right\|<M_{\psi}, \forall z \notin S_{\psi / 2}$. For any $w \notin S_{\psi}, \exists \sqrt{w}$ such that both $\sqrt{w}$ and $-\sqrt{w}$ are not in $S_{\psi / 2}$, thus $\left\|\left(w-A^{2}\right)^{-1}\right\| \leq\left\|(\sqrt{w}-A)^{-1}\right\|\left\|(\sqrt{w}+A)^{-1}\right\| \leq M_{\psi}^{2} /|w|$.

THEOREM 3.7. ([17]) Suppose $A$ is of type $\theta<\pi / 2$ and $\operatorname{Im}(A)$ is dense. Then $A$ is injective and $A^{-1}$ is of type $\theta$.

For operators $A$ of type $\theta<\pi$, fractional powers $A^{\alpha}$, for $0<\alpha<1$, are defined in Balakrishnan [4], by taking the closure of the following operator:

$$
A^{\alpha} x \equiv \frac{\sin \alpha \pi}{\alpha \pi} \int_{0}^{\infty}(A+t)^{-1} A x d\left(t^{\alpha}\right), \quad x \in \mathscr{D}(A),
$$

or, if $\theta<\pi / 2$, also by the Bochner-Phillips calculus [8, 38, 49, 50, 30, 44, 37]; these constructions are equal. Formula (3.8) also defines fractional powers of $A$ for complex $\alpha$ whose real part is between 0 and 1 . (See also $[21,27,31,32,33$, 45]).

The following, from [27], illustrates the sort of spectral intuition one expects; see also [44, Proposition 2.3.2].

LemMa 3.9. Suppose $A$ is of type $\theta<\pi$ and $0<\alpha<1$. Then $A^{\alpha}$ is of type $\alpha \theta$.

In particular, if $-A$ generates a strongly continuous bounded semigroup, then $-A^{\alpha}$ generates a bounded strongly continuous holomorphic semigroup of angle $\pi / 2(1-\alpha)$.

For $\alpha>1, A^{\alpha}$ is defined to be the closure of $A^{\alpha} x \equiv A^{\alpha-n} A^{n} x$, for $n<\alpha<$ $n+1, x \in D\left(A^{n}\right)$. (See [21].)

By writing $A^{r}=\left(A^{\frac{r}{n}}\right)^{n}$, for $n \in \mathbb{N}, n>r$, Lemma 3.9 and Corollary 3.6 immediately yield the following.

COROLlary 3.10. Suppose $A$ is of type $\theta<\pi$ and $r \theta<\pi$. Then $A^{r}$ is of type $r$. 
For example, $-\triangle^{r}$ generates a bounded holomorphic semigroup of angle $\pi / 2, \forall r>0$, on $L^{p}\left(\mathbb{R}^{m}\right)(1 \leq p<\infty)$, where $\triangle$ is the Laplacian.

\section{Some new results on fractional powers of operators and the Bochner-Phillips functional calculus}

Fractional powers have the important multiplicative property

$$
\left(A^{\alpha}\right)^{\beta}=A^{\alpha \beta},
$$

when $0<\alpha<1,0<\beta<1([45,33,50])$.

If $f_{r}(z) \equiv z^{r}$, for $r>0$, and the fractional powers are defined by the Bochner-Phillips functional calculus, then (4.1) may be written as

$$
f_{\beta} \circ f_{\alpha}(A)=f_{\beta}\left(f_{\alpha}(A)\right),
$$

when $0<\alpha<1,0<\beta<1$ (note that $f_{\beta}$ and $f_{\alpha}$ are in $\mathscr{B}$ ).

We shall now prove this multiplicative property for all functions in $\mathscr{B}$.

THEOREM 4.2. (a) $\mathscr{B}$ is closed under composition, that is, $f \circ g \in \mathscr{B}$ whenever $f$ and $g$ are in $\mathscr{B}$. (See [7].)

(b) If $-A$ generates a bounded strongly continuous semigroup, then $(f \circ g)(A)=f(g(A)), \forall f, g \in \mathscr{B}$.

The multiplicative identity (4.1) can easily be extended to $\beta>1([4,33])$. What is of much more interest is to extend (4.1) to the case when $\alpha>1$; in particular, to show that

$$
\left(A^{n}\right)^{1 / n}=A, \quad \text { for } n \in \mathbb{N},
$$

because this is a result about uniqueness of the fractional powers (see [33]). It is well-known that (4.3) is false, in general. Take $A \equiv i d / d x$, on $L^{2}(\mathbb{R})$, then $\left(A^{2}\right)^{\frac{1}{2}} \neq A$ (see $\left.[49,50,21,22]\right)$. The explanation here is simple, even for real numbers: $\sqrt{a^{2}}=|a| \neq a$, unless $a>0$. The operator $i d / d x$ has its spectrum on both sides of the origin. We shall prove that, if $A$ is of type $\theta<\pi$, then

$$
\left(A^{r}\right)^{1 / r}=A, \quad \text { when } r \theta<\pi .
$$

(Proposition 4.7). More generally, we will consider the composition of Bernstein functions with fractional powers. 
THEOREM 4.5. Suppose $r>0, A$ is an operator of type $\theta<\pi / 2$ and $r \theta<\pi / 2$.

Let $f_{r}(z) \equiv z^{r}$ and suppose that both $f$ and $f \circ f_{r}$ are in $\mathscr{B}$. Then

$$
f\left(A^{r}\right)=\left(f \circ f_{r}\right)(A) .
$$

(Note that $A^{r}$ is of type $\theta$, by Corollary 3.10.)

Proposition 4.7. ([29, Theorem 10.6]) Suppose $A$ is of type $\theta<\pi$, $0<\alpha r \theta<\pi, 0<r \theta<\pi$. Then

$$
\left(A^{r}\right)^{\alpha}=A^{r \alpha} .
$$

See also Theorem 5.18 below.

The following proposition and corollary show that, as a map $A \mapsto f(A)$, the Bochner-Phillips functional calculus is continuous, in a manner analogous to the strong operator topology.

Proposition 4.8. Suppose $-A$ and $-B$ generate commuting bounded strongly continuous semigroups. Then, for any $f \in \mathscr{B}, f$ as in Theorem 2.1(iii), $s>0$,

$$
\|f(A) x-f(B) x\| \leq\|A x-B x\|\left(a+M^{2} \int_{0}^{s} d \sigma(t)\right)+2 M \int_{s}^{\infty} \frac{d \sigma(t)}{t},
$$

when $x \in \mathscr{D}(A) \cap \mathscr{D}(B)$, where $\left\|e^{-t A}\right\| \leq M,\left\|e^{-t B}\right\| \leq M, \forall t \geq 0$.

COROLLARY 4.10. Suppose $\left\{-A_{k}\right\}_{k=0}^{\infty}$ is a sequence of operators that generate commuting strongly continuous semigroups and $\exists M<\infty$ such that $\left\|e^{-t A_{k}}\right\| \leq M, \forall t \geq 0, k \in \mathbb{N}$. Suppose $x \in \bigcap_{k=0}^{\infty} \mathscr{D}\left(A_{k}\right)$ such that $\lim _{k \rightarrow \infty} A_{k} x=A_{0} x$. Then

$$
\lim _{k \rightarrow \infty} f\left(A_{k}\right) x=f\left(A_{0}\right) x, \quad \forall f \in \mathscr{B} .
$$

It is of interest that, when $\varepsilon-A$ generates a bounded strongly continuous semigroup, for some $\varepsilon>0$, the Bochner-Phillips functional calculus may be written as an unbounded Cauchy integral, as with the well-known RieszDunford functional calculus (see [19]). We will use this representation to prove Theorem 4.5, as well as some subsequent theorems. 
THEOREM 4.11. Suppose $\varepsilon-A$ generates a bounded strongly continuous semigroup, for some $\varepsilon>0$. Then, for any $f \in \mathscr{B}$,

$$
\begin{aligned}
& e^{-t f(A)} x=\int_{\Gamma_{\varepsilon}} e^{-t f(w)}(w-A)^{-1} A^{k} x \frac{d w}{2 \pi i w^{k}}\left(k \geq 2, x \in \mathscr{D}\left(A^{k}\right)\right) \\
& f(A) x=\int_{\Gamma_{\varepsilon}} f(w)(w-A)^{-1} A^{k} x \frac{d w}{2 \pi i w^{k}}\left(k \geq 3, x \in \mathscr{D}\left(A^{k}\right)\right)
\end{aligned}
$$

where $\Gamma_{\varepsilon} \equiv$ the boundary of $R_{\varepsilon} \equiv\{z \mid \operatorname{Re}(z)>\varepsilon\}$, for $\varepsilon$ sufficiently small.

ProOF OF THEOREM 4.2. (a). One finds by induction that

$$
(-1)^{n}(f \circ g)^{(n+1)}(t) \geq 0, \forall t>0, n=0,1,2, \ldots,
$$

in the same way as in the proof of (i) of Theorem 2.1 in $[9$, p. 83].

(b). We will show that

$$
\sigma_{f \circ g}^{t}(w)=\int_{0}^{\infty} \sigma_{g}^{s}(w) d \sigma_{f}^{t}(s)
$$

$\forall w, t \geq 0, f, g \in \mathscr{B}$, where $\sigma_{f}$ is as in Theorem 2.1(iii). The calculation follows, for $x>0$.

$$
\begin{aligned}
e^{-t(f \circ g)(x)} & =\int_{0}^{\infty} e^{-s g(x)} d \sigma_{f}^{t}(s) \\
& =\int_{0}^{\infty}\left(\int_{0}^{\infty} e^{-w x} d \sigma_{g}^{s}(w)\right) d \sigma_{f}^{t}(s) \\
& =\int_{0}^{\infty} e^{-w x} d\left(\int_{0}^{\infty} \sigma_{g}^{s}(w) d \sigma_{f}^{t}(s)\right),
\end{aligned}
$$

by a Fubini type argument (see [3]).

Equation $\left(*^{\prime}\right)$ now follows from the uniqueness of the representation in Theorem 2.1(iii).

Now suppose $-A$ generates a bounded strongly continuous semigroup. For $t \geq 0$, we make a similar calculation, using $\left(*^{\prime}\right)$.

$$
\begin{aligned}
e^{-t f(g(A))} & =\int_{0}^{\infty} e^{-s g(A)} d \sigma_{f}^{t}(s) \\
& =\int_{0}^{\infty}\left(\int_{0}^{\infty} e^{-w A} d \sigma_{g}^{s}(w)\right) d \sigma_{f}^{t}(s)
\end{aligned}
$$




$$
\begin{aligned}
& =\int_{0}^{\infty} e^{-w A} d\left(\int_{0}^{\infty} \sigma_{g}^{s}(w) d \sigma_{f}^{t}(s)\right) \\
& =\int_{0}^{\infty} e^{-w A} d \sigma_{f \circ g}^{t}(w) \\
& =e^{-t(f \circ g)(A)},
\end{aligned}
$$

so that $f(g(A))=(f \circ g)(A)$.

PROOF OF PROPOSITION 4.8.

$$
\begin{aligned}
\left\|e^{-t B} x-e^{-t A} x\right\| & =\left\|\int_{0}^{t} e^{-(t-s) A} e^{-s B}(A x-B x) d s\right\| \\
& \leq t M^{2}\|A x-B x\|
\end{aligned}
$$

Thus,

$$
\begin{aligned}
\|f(A) x-f(B) x\| & \leq a\|A x-B x\|+\int_{0}^{\infty}\left\|e^{-t B} x-e^{-t A} x\right\| \frac{d \sigma(t)}{t} \\
& \leq a\|A x-B x\|+\int_{0}^{s} M^{2}\|A x-B x\| d \sigma(t) \\
& +2 M \int_{s}^{\infty} \frac{d \sigma(t)}{t},
\end{aligned}
$$

which implies (4.9).

PROOF OF COROLlARY 4.10. It is clear from Proposition 4.8 that $\overline{\lim }_{k \rightarrow \infty}$ $\left\|f\left(A_{k}\right) x-f\left(A_{0}\right) x\right\| \leq 2 M \int_{s}^{\infty} d \sigma(t) / t, \forall s>0$. Since $\int_{1}^{\infty} d \sigma(t) / t$ is finite, (see Theorem 2.1(iii)), this implies that $\lim _{k \rightarrow \infty}\left\|f\left(A_{k}\right) x-f\left(A_{0}\right) x\right\|=0$, as desired.

PROOF OF THEOREM 4.11. By Theorem (2.4), calculating as with the RieszDunford functional calculus,

$$
\begin{aligned}
e^{-t f(A)} x & =\int_{0}^{\infty} e^{-s A} A^{-k} A^{k} x d \sigma^{t}(s) \\
& =\int_{0}^{\infty} \int_{\Gamma_{\varepsilon}} e^{-s w}(w-A)^{-1} A^{k} x \frac{d w}{2 \pi i w^{k}} d \sigma^{t}(s) \\
& =\int_{\Gamma_{\varepsilon}}\left[\int_{0}^{\infty} e^{-s w} d \sigma^{t}(s)\right](w-A)^{-1} A^{k} x \frac{d w}{2 \pi i w^{k}}
\end{aligned}
$$


so that (4.12) follows from Theorem 2.1. For the first equality above, we applied the Riesz-Dunford calculus, as in [19, part I, VII.9]. Note that interchanging the order of integration is justified by the fact that $\left\|(w-A)^{-1}\right\|$ is bounded on $\Gamma_{\varepsilon}$, since $\varepsilon-A$ generates a bounded strongly continuous semigroup.

Since $\sup _{|w| \rightarrow \infty}|f(w) / w|$ is finite, for any $f \in \mathscr{B}$, we may obtain (4.13) from (4.12) by differentiating at $t=0$.

PROOF OF THEOREM 4.5. First, assume $0 \in \rho(A)$.

Let $S_{\psi, \varepsilon} \equiv\{z|| \arg (z) \mid<\psi\} \cap\{z|| z \mid>\varepsilon\}, \Gamma_{\psi, \varepsilon}$ be the boundary of $S_{\psi, \varepsilon}$. Choosing $\phi$, $\psi$ so that $0<r \theta<r \phi<\psi<\pi / 2$, we may argue exactly as in the proof of Theorem 4.11 to show that

$$
f\left(A^{r}\right) x=\int_{\Gamma_{\psi, \varepsilon}} f(w)\left(w-A^{r}\right)^{-1} A^{3} x \frac{d w}{2 \pi i w^{3}} .
$$

By the Riesz-Dunford functional calculus, as in [19, part I, VII.9], we have, for $w \notin S_{\phi, \varepsilon}$,

$$
\left(w-A^{r}\right)^{-1} x=\int_{\Gamma_{\phi, \varepsilon}}\left(w-z^{r}\right)^{-1}(z-A)^{-1} x \frac{d z}{2 \pi i},
$$

This agrees with the definition of $A^{r}$; the proof is the same as that of [44, Lemma 2.3.2].

We now apply $(*)$ and $(* *)$, for $x \in \mathscr{D}\left(A^{3}\right)$, as follows, where all integrals are taken over $\Gamma_{\psi, \varepsilon}$, for $\varepsilon>0$ sufficiently small.

$$
\begin{aligned}
f\left(A^{r}\right) x & =\int f(w)\left(w-A^{r}\right)^{-1} A^{3} x \frac{d w}{2 \pi i w^{3}} \\
& =\int f(w) \int\left(w-z^{r}\right)^{-1}(z-A)^{-1} A^{3} x \frac{d z}{2 \pi i} \frac{d w}{2 \pi i w^{3}} \\
& =\int\left[\int f(w)\left(w-z^{r}\right)^{-1} \frac{d w}{2 \pi i w^{3}}\right](z-A)^{-1} A^{3} x \frac{d z}{2 \pi i} \\
& =\int f\left(z^{r}\right)(z-A)^{-1} A^{3} x \frac{d z}{2 \pi i z^{3}}=\left(f \circ f_{r}\right)(A) x .
\end{aligned}
$$

It is clear from Theorem 2.2 that $\mathscr{D}\left(A^{3}\right)$ is left invariant by $e^{-\operatorname{tg}(A)}, \forall g \in \mathscr{B}$. This implies that $\mathscr{D}\left(A^{3}\right)$ is a core for $g(A)$. Thus the calculation above implies (4.6), when $0 \in \rho(A)$.

For general $A$ as in the Theorem, we may now assert that

$(* * *)$

$$
f\left((A+\varepsilon)^{r}\right)=\left(f \circ f_{r}\right)(A+\varepsilon), \quad \forall \varepsilon>0 .
$$


It is not hard to see, from (3.8), that $\lim _{\varepsilon \rightarrow 0}(A+\varepsilon)^{r} x=A^{r} x, \forall x \in \mathscr{D}(A)$. Thus, by Corollary 4.10, and $(* * *), f\left(A^{r}\right) x=\left(f \circ f_{r}\right)(A) x, \forall x \in \mathscr{D}(A)$; as argued earlier, this implies that $f\left(A^{r}\right)=\left(f \circ f_{r}\right)(A)$.

PROOF OF PROPOSITION 4.7. This is exactly the same as the proof of Theorem 4.5 , using the fact that

$$
A^{r} x=\int_{\Gamma_{\psi, \varepsilon}} w^{r}(w-A)^{-1} A^{k} x \frac{d w}{2 \pi i w^{k}},
$$

for $k$ sufficiently large, $x \in \mathscr{D}(A)$, when $0 \in \rho(A), A$ is of type $\theta<\pi$ and $\theta<\psi<\pi$.

\section{A functional calculus construction for Stieltjes functions and nonnegative operator monotone functions}

We shall consider a large class of functions that we will show preserve type, that is, $f(A)$ is of type $\theta$ whenever $A$ is, so that $-f(A)$ generates a bounded holomorphic semigroup of angle $\phi$ whenever $-A$ does (see Section 3 ).

The class $\mathscr{R}$ consists of all nontrivial functions of the form

$$
f(z)=a z+b+\int_{0}^{\infty}(z /(z+t)) d \mu(t), \quad z \in \mathbf{C}-(-\infty, 0),
$$

where $a, b \geq 0$ are constants and $\mu$ is a nondecreasing function on $[0, \infty)$ such that $\int_{0}^{\infty} d \mu(t) /(1+t)<\infty$. We assume that $\mu$ is normalized as follows: $\mu(0)=0$ and $\mu(t)=\mu\left(t^{+}\right), \forall t \geq 0$. With this normalization, $a, b$ and $\mu$ are uniquely determined by $f: a=\lim _{t \rightarrow \infty} f(t) / t, b=f(0) . \mathscr{R} \subseteq \mathscr{B}$, that is, the restriction of $f \in \mathscr{R}$ to $\mathbb{R}^{+}$is in $\mathscr{B}$.

In the terminology of $[18,2,23,35]$, functions in $\mathscr{R}$ are nonnegative operator monotone functions on $\mathbb{R}^{+}$. They have the following very useful geometric characterization, where we write $U H P$ for the upper half plane $\{z \in \mathbf{C} \mid \operatorname{Im}(z)>$ $0\}$.

LEMMA 5.2. A function $f$ defined and continuous on $\mathbf{C}-(-\infty, 0)$ and holomorphic in the interior belongs to $\mathscr{R}$ if and only if $f: U H P \rightarrow U H P$ and $f:(0, \infty) \rightarrow(0, \infty)$.

The "only if" part is obvious, as $\operatorname{Im}(z /(z+t))=t \operatorname{Im}(z) /|z+t|^{2}$. Functions in $\mathscr{R}$ also preserve the lower half plane $L H P$. The "if" part follows from 
a classical integral representation for functions preserving $U H P$ obtained by Nevanlinna; see [18, 23, 35].

We consider also the class $\mathscr{S}$ of Stieltjes functions

$$
g(z)=a+\int_{0}^{\infty} \frac{1}{z+t} d \lambda(t), \quad z \in \mathbf{C}-(-\infty, 0],
$$

where $a \geq 0$, and $\lambda$ is right-continuous and nondecreasing and where $\int_{0}^{\infty} d \lambda(t) /(1+t)<\infty$. The Stieltjes functions are characterized by the property that they interchange $U H P$ and $L H P$.

LEMMA 5.4. A holomorphic function $f$ on $\mathbf{C}-(-\infty, 0]$ belongs to $\mathscr{S}$ if and only if $f: U H P \rightarrow L H P$ and $f:(0, \infty) \rightarrow(0, \infty)$.

The "only if" follows from the fact that $\operatorname{Im}(1 /(z+t))=-\operatorname{Im}(z) /|z+t|^{2}$; for the "if" part see [1, p. 127] and [5, 35].

The following properties, which follow immediately from Lemmas 5.3 and 5.4 and $(5.1),(5.3)$, may be found in $[5,35]$.

LEMMA 5.5. (i) The class $\mathscr{R}$ is closed under composition, that is, $f \circ g$ is in $\mathscr{R}$ whenever $f$ and $g$ are in $\mathscr{R}$.

(ii) $f \circ g$ and $g \circ f \in \mathscr{S}$ whenever $f \in \mathscr{R}$ and $g \in \mathscr{S}$.

(iii) If $f$ and $g$ are in $\mathscr{S}$ and $f \circ g$ is defined and continuous at 0 , then $f \circ g \in \mathscr{R}$.

(iv) The classes $\mathscr{R}$ and $\mathscr{S}$ are closed convex cones which are logarithmically convex.

(v) If $g \in \mathscr{S}$, then $g /(\lambda g+1) \in \mathscr{S}, \forall \lambda \geq 0$.

(vi) $z \mapsto z g(z) \in \mathscr{R}$ whenever $g \in \mathscr{S}$.

(vii) $z \mapsto f(z) / z \in \mathscr{S}$ whenever $f \in \mathscr{R}$.

A typical representative of $\mathscr{S}$ is the function $g(z) \equiv 1 / z$. The properties (ii), (iii) and (iv) above imply that

COROllaRy 5.6. (i) $f \in \mathscr{R} \leftrightarrow 1 / f \in \mathscr{S}$, if $f$ is nontrivial.

(ii) If $f \in \mathscr{R}$, then $z \mapsto f(1 / z) \in \mathscr{S}$ and $z \mapsto z f(1 / z) \in \mathscr{R}$.

(iii) $z \mapsto\left[f\left(z^{-1}\right)\right]^{-1} \in \mathscr{R}$ whenever $f$ is a nontrivial member of $\mathscr{R}$.

A fundamental connection between the classes $\mathscr{R}$ and $\mathscr{S}$ is

LEMMA 5.7. $g \in \mathscr{S} \leftrightarrow \exists f_{g} \in \mathscr{R}$ such that $g(z)=f_{g}(1 / z)$. 
This follows from the definition of $\mathscr{S}$ and $\mathscr{R}$ : if $g$ is given by (5.3), we take

$$
f_{g}(w)=a+\int_{0}^{\infty} \frac{w}{1+t w} d \lambda(t)
$$

Note that the functions in both $\mathscr{R}$ and $\mathscr{S}$ preserve the right half-plane.

EXAMPLES 5.9. Some examples of functions in $\mathscr{R}$ are
(a) $z^{\alpha}=\sin (\alpha \pi) / \alpha \pi \int_{0}^{\infty} z /(z+t) d\left(t^{\alpha}\right), 0<\alpha<1$;
(b) $\log (1+z)=\int_{1}^{\infty} z /(z+t) d(\log t)$;
(c) $\sqrt{z} \arctan (\beta / \sqrt{z})=\int_{0}^{\beta^{2}} z /(z+t) d(\sqrt{t}), \beta>0$.

The properties in 5.5 and 5.6 enable us to make new examples from the examples above.

DEFINITION 5.10. Suppose $A$ is of type $\theta<\pi$ and $f \in \mathscr{R}$ is as in (5.1). We define $f(A)$ to be the closure of

(5.11) $f(A) x \equiv a A x+b x+\int_{0}^{\infty}(t+A)^{-1} A x d \mu(t), \quad x \in \mathscr{D}(A)$.

This is well-defined, since $\left\|t(t+A)^{-1}\right\|$ is bounded, as $t \rightarrow \infty$. This boundedness also implies that the operator defined by (5.11) is closable, for the following reason. Let $M \equiv \sup \left\{\left\|s(s+A)^{-1}\right\| \mid s>0\right\}$. Then, for any $x \in \mathscr{D}(A)$,

$$
\begin{aligned}
(A+1)^{-1} f(A) x= & a A(A+1)^{-1} x+b(A+1)^{-1} x \\
& +\int_{0}^{1}\left(1-t(A+t)^{-1}\right)(A+1)^{-1} x d \mu(t) \\
& +\int_{1}^{\infty}(t+A)^{-1} A(A+1)^{-1} x d \mu(t),
\end{aligned}
$$

so that

$$
\begin{aligned}
& \left\|(A+1)^{-1} f(A) x\right\| \\
& \quad \leq\left(a(M+1)+b M+(M+1) \int_{0}^{1} d \mu(t)+M(M+1) \int_{1}^{\infty} \frac{d \mu(t)}{t}\right)\|x\| .
\end{aligned}
$$

By the comments after $(5.1)$, this implies that $(A+1)^{-1} f(A)$ is bounded, so that, since $A$ is closed, the operator defined by (5.11) is closable. 
We shall write $f(A)$ for the closure of the operator defined by (5.11). In explicit computations, however, we shall use (5.11). Such a calculation was considered also by Hirsch $([25,26])$.

Since $(t+A)^{-1}=\left(1+t A^{-1}\right)^{-1} A^{-1}$, we may similarly define a functional calculus for $g \in \mathscr{S}$. (See Theorem 3.7.)

DEFINITION 5.12. Suppose $A$ is of type $\theta<\pi, \operatorname{Im}(A)$ is dense and $g \in \mathscr{S}$ is as in (5.3). We define $g(A)$ to be the closure of

$$
g(A) x \equiv a x+\int_{0}^{\infty}(t+A)^{-1} x d \lambda(t), \quad x \in \operatorname{Im}(A) .
$$

Since $\mathscr{R} \subseteq \mathscr{B}, f(A)$ is also defined in Theorem 2.2, for $f \in \mathscr{R}$. We will show later that these two constructions agree (Proposition 5.17). Our construction (5.11) has some advantages. It involves only resolvents, which are much easier to calculate explicitly than semigroups $\left\{e^{-t A}\right\}$. Definition (5.11) makes sense for a wider class of operators, operators of type $\theta<\pi$. Lemma 5.7 allows us to consider the Stieltjes functional calculus via $g(A)=f_{g}\left(A^{-1}\right)$, for $g \in \mathscr{S}$.

As a map $A \mapsto f(A)$, the functional calculus (5.11) is continuous, in a sense analogous to the strong operator topology; this is expressed precisely in Proposition 5.14 and Corollary 5.16.

Proposition 5.14. Suppose $A$ and $B$ are two operators of type $(\theta, M)$, where $\theta<\pi$, whose resolvents commute. Then $\forall x \in \mathscr{D}(A) \cap \mathscr{D}(B), s>0$,

$$
\|f(A) x-f(B) x\| \leq 2 M\|x\| \mu(s)+\|A x-B x\|\left(a+M^{2} \int_{s}^{\infty} \frac{d \mu(t)}{t}\right),
$$

for any $f \in \mathscr{R}$ as in (5.1).

Proof. Since $(A+t)^{-1} A x=x-t(A+t)^{-1} x$, we have

$$
\begin{gathered}
\|f(A) x-f(B) x\| \leq a\|A x-B x\|+\int_{0}^{\infty}\left\|t(B+t)^{-1} x-t(A+t)^{-1} x\right\| d \mu(t) \\
=a\|A x-B x\|+\int_{0}^{s}\left\|t(B+t)^{-1} x-t(A+t)^{-1} x\right\| d \mu(t) \\
\quad+\int_{s}^{\infty} t\left\|(A+t)^{-1}(B+t)^{-1}(A x-B x)\right\| d \mu(t) .
\end{gathered}
$$

Equation (5.15) now follows immediately from the fact that $\left\|t(A+t)^{-1}\right\|$ and $\left\|t(B+t)^{-1}\right\|$ are less than or equal to $M, \forall t \geq 0$ (see Definition 3.3). 
COROLLARY 5.16. Suppose $\left\{A_{k}\right\}_{k=0}^{\infty}$ is a sequence of operators of type $(\theta, M)$, whose resolvents commute, $x \in \bigcap_{k=0}^{\infty} \mathscr{D}\left(A_{k}\right)$ and $\lim _{k \rightarrow \infty} A_{k} x=A_{0} x$. Then $\lim _{k \rightarrow \infty} f\left(A_{k}\right) x=f\left(A_{0}\right) x, \forall f \in \mathscr{R}$.

PROOF. It is clear from Proposition 5.14 that $\varlimsup_{k \rightarrow \infty}\left\|f\left(A_{k}\right) x-f\left(A_{0}\right) x\right\| \leq$ $2 M\|x\| \mu(s), \forall s>0$. Since $\lim _{s \rightarrow 0} \mu(s)=0$ (see the comments after Theorem (5.1)), this implies that $\lim _{k \rightarrow \infty}\left\|f\left(A_{k}\right) x-f\left(A_{0}\right) x\right\|=0$, as desired.

We must verify that our construction, in Definition 5.10 , agrees with the Bochner-Phillips construction, when $f \in \mathscr{R}$. We do this essentially by showing that they both agree with the Cauchy integral formula construction (4.13).

PROPOSITION 5.17. Suppose $f \in \mathscr{R}$ and $-A$ generates a bounded strongly continuous semigroup. Let $f(A)_{1}$ be the operator from Definition 5.10 and let $f(A)_{2}$ be the operator defined by Theorem 2.2. Then $f(A)_{1}=f(A)_{2}$.

PROOF. First, suppose $\varepsilon-A$ generates a bounded strongly continuous semigroup and $x \in \mathscr{D}\left(A^{3}\right)$. Then, with $\Gamma_{\varepsilon}$ as in Theorem 4.11, we argue as with the Riesz-Dunford functional calculus,

$$
\begin{aligned}
2 \pi i f(A)_{2} x & =\int_{\Gamma_{\varepsilon}} f(w)(w-A)^{-1} A^{3} x \frac{d w}{w^{3}} \\
& =\int_{\Gamma_{\varepsilon}}\left[a w+b+\int_{0}^{\infty} \frac{w}{w+t} d \mu(t)\right](w-A)^{-1} A^{3} x \frac{d w}{w^{3}} \\
& =a A x+b x+\int_{0}^{\infty}\left[\int_{\Gamma_{\varepsilon}} \frac{w}{w+t}(w-A)^{-1} A^{3} x \frac{d w}{w^{3}}\right] d \mu(t) \\
& =2 \pi i \int_{0}^{\infty}(t+A)^{-1} A x d \mu(t) \\
& =2 \pi i f(A)_{1} x .
\end{aligned}
$$

Now suppose $-A$ generates a bounded strongly continuous semigroup. For $x \in \mathscr{D}\left(A^{3}\right)$, the calculation above shows that $f(A+\varepsilon)_{1} x=f(A+\varepsilon)_{2} x, \forall \varepsilon>0$. Corollaries 4.10 and 5.16 now imply that

$$
f(A)_{1} x=f(A)_{2} x, \forall x \in \mathscr{D}\left(A^{3}\right) .
$$

It is clear from (2.4) that $e^{-t f(A)_{2}}$ leaves $\mathscr{D}\left(A^{3}\right)$ invariant. This implies that $\mathscr{D}\left(A^{3}\right)$ is a core for $f(A)_{2}$, thus, since $f(A)_{1}$ is closed, (*) implies that $f(A)_{2} \subseteq$ 
$f(A)_{1}$. Since, in an equivalent norm, $f(A)_{1}$ is accretive and $f(A)_{2}$ is $m$ accretive, this implies that $f(A)_{1}=f(A)_{2}$.

We have also the following analogue of Theorem 4.5, and extension of Proposition 4.7.

THEOREM 5.19. Suppose $r>0, A$ is an operator of type $\theta<\pi$ and $r \theta<\pi$. Let $f_{r}(z) \equiv z^{r}$ and suppose that both $f$ and $f \circ f_{r}$ are in $\mathscr{R}(\mathscr{S})$. Then

$$
f\left(A^{r}\right)=\left(f \circ f_{r}\right)(A) .
$$

The proof is the same as that of Theorem 4.5 , with the representation

$$
\left(A^{r}+t\right)^{-1} A^{r} x=\int_{\Gamma_{\psi, \varepsilon}}\left(w^{r}+t\right)^{-1} w^{r}(w-A)^{-1} A^{k} x \frac{d w}{2 \pi i w^{k}},
$$

for appropriate $\psi, \varepsilon, k$ (cf. [29, p. 322])

\section{Preservation of type}

We prove that, when $-A$ generates a bounded holomorphic semigroup of angle $\theta(0<\theta \leq \pi / 2)$, then the same is true of $f(A)$, for any $f \in \mathscr{R}+\mathscr{S}$. Thus we are able to generate new generators from a given one.

In terms of Definition 3.3, this is saying that functions in $\mathscr{R}+\mathscr{S}$ preserve type, that is, $f(A)$ is of type $\theta$ whenever $A$ is. (Theorem 6.4.)

More generally, we obtain what is more than a spectral mapping theorem, what may be called a type mapping theorem: if $h \in \mathscr{R}$ and $h: S_{\pi} \rightarrow S_{\alpha \pi}$, for some $0<\alpha<1$, and $A$ is of type $\theta$, then $h(A)$ is of type $\alpha \theta$. (Corollary 6.3.) This is an extension of Kato's lemma (Lemma 3.9).

THEOREM 6.1. Suppose $f \in \mathscr{R}, 0 \leq \theta<\pi / 2$ and $A$ is of type $\theta$. Then $f(A)$ is of type $\theta$.

REMARK 6.2. It will be clear from the proof that the conclusion of Theorem 6.1 is valid whenever $f \in \mathscr{B}$ has the property that the map $z \mapsto$ $\left[f\left(z^{1 / r}\right)\right]^{r} \in \mathscr{B}, \forall r>1$. It is thus natural to ask which functions in $\mathscr{B}$ have this property. It is straightforward to verify that functions in $\mathscr{R}$ have this property (Lemma 6.6); it is an open question whether all functions in $\mathscr{B}$ have this property. 
COROLlary 6.3. Suppose $0 \leq \theta<\pi / 2$, A is of type $\theta, h \in \mathscr{R}$ and $h: S_{\pi} \rightarrow$ $S_{\alpha \pi}$, for some $0<\alpha<1$. Then $h(A)$ is of type $\alpha \theta$.

When the range of $A$ is dense, we can prove that type is preserved by a large class of functions, $\mathscr{R}+\mathscr{S}$; this includes functions not in $\mathscr{B}$.

For $k=f+g$, where $f \in \mathscr{R}$ and $g \in \mathscr{S}$, we define $k(A)$ to be the closure of $f(A)+g(A)$, with domain $\mathscr{D}(f(A)) \cap \mathscr{D}(g(A))$. The operator $-k(A)$ is then the generator of $e^{-t f(A)} e^{-t g(A)}$.

THEOREM 6.4. Suppose $0 \leq \theta<\pi / 2, A$ is of type $\theta, \operatorname{Im}(A)$ is dense and $k \in \mathscr{R}+\mathscr{S}$. Then $k(A)$ is of type $\theta$.

THEOREM 6.5. The functions in $\mathscr{R}$ preserve all sectors $S_{\psi}, 0 \leq \psi \leq \pi$.

PROOF. A brief calculation shows that $\phi_{t}(z) \equiv z /(z+t)$ preserves sectors, $\forall t \geq 0$. Thus this follows from the representation (5.1).

LEMMA 6.6. Suppose $f \in \mathscr{R}$ and $r>1$. Then $f_{r}(z) \equiv\left[f\left(z^{1 / r}\right)\right]^{r}$ is also in $\mathscr{R}$. (cf. [35, Corollary 2]).

PROOF. This follows from the geometric characterization of $\mathscr{R}$, Lemma 5.2 and Lemma 6.5 .

PROOF OF THEOREM 6.1. Fix $r>1$ such that $r \theta<\pi / 2$. Let $h(z) \equiv f\left(z^{1 / r}\right)$, $g(z) \equiv[h(z)]^{r}$. By Lemma 6.6, $g \in \mathscr{R}$; it is clear from Lemma 5.2 that $h \in \mathscr{R}$.

By Corollary 3.10, $A^{r}$ is of type $r \theta$. By Theorem $2.2, g\left(A^{r}\right)$ is of type $\pi / 2$, thus by Corollary 3.10 again,

$$
\begin{aligned}
f(A) & =f\left(\left(A^{r}\right)^{1 / r}\right), \text { by Proposition } 4.7, \\
& =h\left(A^{r}\right), \text { by Theorem } 4.5, \\
& =\left[g\left(A^{r}\right)\right]^{1 / r}, \text { by Theorem } 4.2,
\end{aligned}
$$

is of type $\pi / 2 r$. This implies that, $\forall \psi>\theta, f(A)$ is of type $\psi$, which is equivalent to $f(A)$ being of type $\theta$.

PROOF OF COROLlaRY 6.3. $g(z) \equiv[h(z)]^{\frac{1}{\alpha}} \in \mathscr{R}$, by Lemma 6.6. By Theorem 6.1, $g(A)$ is of type $\theta$. By Theorem $4.2, h(A)=[g(A)]^{\alpha}$, thus by Lemma $3.9, h(A)$ is of type $\alpha \theta$. 
ProOF OF THEOREM 6.4. By Theorem 6.1, since the sum of two commuting operators of type $\theta$ is of type $\theta$, it is sufficient to show that $g(A)$ is of type $\theta$, whenever $g \in \mathscr{S}$.

By Theorem 3.7, $A$ is injective and $A^{-1}$ is of type $\theta$. For $x \in \mathscr{D}\left(A^{-1}\right)=$ $\operatorname{Im}(A),($ see $(5.3))$

$$
\begin{aligned}
g(A) x & =a x+\int_{0}^{\infty}(t+A)^{-1} x d \lambda(t) \\
& =a x+\int_{0}^{\infty}\left(t A^{-1}+1\right) A^{-1} x d \lambda(t) \\
& =f_{g}\left(A^{-1}\right) x
\end{aligned}
$$

where $f_{g} \in \mathscr{R}$ is given by (5.8). Taking closures, this implies that $g(A)=$ $f_{g}\left(A^{-1}\right)$, which is of type $\theta$, by Theorem 6.1 .

\section{Operators of type 0}

We show that $f(A)$ is of type 0 , whenever $A$ is of type 0 and $f \in \mathscr{B}$, that is, $-f(A)$ generates a bounded holomorphic strongly continuous semigroup of angle $\pi / 2$. (Theorem 7.2.)

This is a partial answer to the following open question in [28]: suppose $-A$ generates a bounded holomorphic strongly continuous semigroup and $f \in$ $\mathscr{B}$. Does $-f(A)$ also generate a bounded holomorphic strongly continuous semigroup? Theorem 7.2 answers this question in the affirmative, when the angle of the holomorphic semigroup generated by $-A$ is $\pi / 2$; in addition, this angle is preserved by the holomorphic semigroup generated by $-f(A)$ These are the holomorphic semigroups of most interest, since they include elliptic operators, such as the Laplacian (see Example 7.3).

More generally, we show that the answer to this open question is affirmative whenever $-A$ generates a bounded holomorphic strongly continuous semigroup of angle greater than $\pi / 4$ (Proposition 7.4). It is not clear if the angle of the semigroup is preserved, for arbitrary $f \in \mathscr{B}$ (see, however, Remark 6.2 and Theorem 6.1).

The proof is essentially the same as the proof of Theorem 6.1 , with the following additional result.

Proposition 7.1. Suppose $f \in \mathscr{B}$ and $n \in \mathbb{N}$. Then $g_{n}(z) \equiv\left[f\left(z^{1 / n}\right)\right]^{n} \in \mathscr{B}$. 
THEOREM 7.2. Suppose $A$ is of type 0 and $f \in \mathscr{B}$. Then $f(A)$ is of type 0 .

EXAMPLE 7.3. Let $A \equiv-\triangle$, on $L^{p}\left(\mathbb{R}^{n}\right)$, for $1 \leq p<\infty$. Then, $\forall f \in \mathscr{B}$, $f(A)$ is of type 0 .

The same is true for $A \equiv-\triangle$, on $L^{p}(\Omega)$, with Dirichlet boundary conditions, for $1 \leq p<\infty$, where $\Omega \subset \mathbb{R}^{n}$ is a region with smooth boundary.

For the case $1<p<\infty$, an $H^{\infty}$-functional calculus, for $-\Delta$, on $L^{p}(\mathbb{R})$, is constructed in [41], and an $H^{\infty}$-functional calculus for $A \equiv-\Delta$, on $L^{p}(\Omega)$, with Dirichlet boundary conditions, for $1 \leq p<\infty$, where $\Omega \subset \mathbb{R}^{n}$ is as above, is constructed in [20].

Theorem 7.2 follows immediately from the following.

PROPOSITION 7.4. Suppose $A$ is of type $\theta, n \in \mathbb{N}$ and $n \theta<\pi / 2$. Then $f(A)$ is of type $\pi / 2 n, \forall f \in \mathscr{B}$.

PROOF OF PROPOSITION 7.1. For $t>0$, we have

$$
g_{n}^{\prime}(t)=f^{\prime}\left(t^{1 / n}\right)\left[\frac{f\left(t^{1 / n}\right)}{t^{1 / n}}\right]^{n-1}=\phi\left(t^{1 / n}\right),
$$

where $\phi(t) \equiv f^{\prime}(t)[f(t) / t]^{n-1}$ is completely monotone because it is the product of the completely monotone functions $f^{\prime}$ and $t \mapsto f(t) / t$. The latter is easily seen to be completely monotone using the integral representation in Theorem 2.1(iii). Since $t \mapsto t^{1 / n}$ is a Bernstein function, it now follows from Theorem 2.1(i) that $g_{n}^{\prime}$ is completely monotone, that is, $g_{n} \in \mathscr{B}$.

PROOF OF PROPOSITION 7.4. Let $\left.h_{n}(z) \equiv\left[g_{n}(z)\right]^{1 / n}\right)$. This is also in $\mathscr{B}$, by Theorem 4.2 and Proposition 7.1. By Corollary 3.10, $A^{n}$ is of type $n \theta$. By Theorem 2.2, $g_{n}\left(A^{n}\right)$ is of type $\pi / 2$, thus by Corollary 3.10 again,

$$
\begin{aligned}
f(A) & =f\left(\left(A^{n}\right)^{1 / n}\right), \quad \text { by Proposition } 4.7, \\
& =h_{n}\left(A^{n}\right), \quad \text { by Theorem } 4.5, \\
& =\left[g_{n}\left(A^{n}\right)\right]^{1 / n}, \quad \text { by Theorem } 4.2,
\end{aligned}
$$

is of type $\pi / 2 n$. 
Added in proof

After the paper was accepted we learned of the following papers which have related results: $[12,39]$

\section{References}

[1] N. Akhiezer, The classical moment problem (Hafner Publishing Company, New York, 1965).

[2] T. Ando, 'Comparison of norms $\||| f(A)-f(B)\| \|$ and $\||f(|A-B|)|\|$ ', Math. Z. 197 (1988), 403-409.

[3] R. B. Ash, Measure, integration and functional analysis (Academic Press, New York, 1972).

[4] A. V. Balakrishnan, 'Fractional powers of closed operators and the semigroups generated by them', Pac. J. Math. 10 (1960), 419-437.

[5] C. Berg, Quelques remarques sur le cone de Stieltjes, Lecture Notes in Math. 814 (Springer, Berlin, 1980) pp. 70-79.

[6] C. Berg, J. P. R. Christensen and P. Ressel, Harmonic Analysis on Semigroups (Springer, Berlin, 1984).

[7] C. Berg and G. Forst, Potential theory on locally compact abelian groups (Springer, Berlin, 1975).

[8] S. Bochner, 'Diffusion equations and stochastic processes', Proc. Nat. Acad. Sci. U.S.A 35 (1949), 368--370.

[9] Harmonic analysis and the theory of probability (University of California Press, Berkeley, 1955).

[10] K. Boyadzhiev and R. deLaubenfels, ' $h h^{\infty}$-functional calculus for perturbations of generators of holomorphic semigroups', Houston J. Math. 17 (1991), 131-147.

[11] O. Bratteli, A. Kishimoto and D. W. Robinson, 'Positivity and monotonicity properties of $C_{0}$ semigroups I', Comm. Math. Phys. 75 (1980), 67-84.

[12] A. S. Carasso and T. Kato, 'On subordinated holomoprhic semigroups', Trans. Amer. Math. Soc. 327 (1991), 867-878.

[13] E. B. Davies, One-parameter semigroups (Academic Press, London, 1980).

[14] R. deLaubenfels, ' $C$-semigroups and the Cauchy problem', J. Funct. Anal, to appear.

[15] _ ' 'Functional calculus and abstract Cauchy problems for operators with polynomially bounded resolvent', J. Funct. Anal., to appear.

[16] - 'Powers of generators of holomorphic semigroups', Proc. Amer. Math. Soc. 99 (1987), 105-108.

[17] — 'Inverses of generators', Proc. Amer. Math. Soc. 104 (1988), 443-448.

[18] W. F. Donoghue, Jr., Monotone matrix functions and analytic continuation (Springer-Verlag, New York, 1974).

[19] N. Dunford and J. T. Schwartz, Linear operators, part I (Interscience, New York, 1958).

[20] X. T. Duong, ' $H^{\infty}$ functional calculus of elliptic operators with $C^{\infty}$ coefficients on $L^{p}$ spaces of smooth domains', J. Austral. Math. Soc. (Series A) 48 (1990), 113-123. 
[21] H. O. Fattorini, The abstract Cauchy problem (Addison Wesley, Reading, 1983).

[22] J. A. Goldstein, Semigroups of operators and applications (Oxford, New York, 1985).

[23] E. Heinz, 'Beitrage zur Störungstheorie der Spektralzerlegung', Math. Ann. 123 (1951), 415-438.

[24] E. Hille and R. S. Phillips, Functional analysis and semigroups (Amer. Math. Soc., Providence, 1957).

[25] F. Hirsch, 'Integrales de resolvantes et calcul symbolique', Ann. Inst. Fourier, Grenoble 22 (4) (1972), 239-264.

[26] - 'Domaines d'operateurs representes comme integrales de resolvantes', J. Funct. Anal. 23 (1976), 199-217.

[27] T. Kato, 'Note on fractional powers of linear operators', Proc. Japan Acad. Ser. A Math. Sci. 36 (1960), 94-96.

[28] A. Kishimoto and D. W. Robinson, 'Subordinate semigroups and order properties', $J$. Austral. Math. Soc. (Series A) 31 (1981), 59-76.

[29] H. Komatsu, 'Fractional powers of operators', Pac. J. Math. 19 (1966), 285-346.

[30] S. G. Krein, Linear differential equations in Banach spaces, Transl. Math. Monographs 29 (Amer. Math. Soc., Providence, 1971).

[31] O. E. Lanford and D. W. Robinson, 'Fractional powers of generators of equicontinuous semigroups and fractional derivatives', J. Austral. Math. Soc. (Series A) 46 (1989), 473504.

[32] C. Martinez and M. Sanz, ' $n^{\text {th }}$ roots of a non-negative operator: conditions for uniqueness', Manuscripta Math. 64 (1989), $403-417$.

[33] C. Martinez, M. Sanz and L. Marco, 'Fractional powers of operators', J. Math. Soc. Japan 40 (1988), 331-347.

[34] A. McIntosh, 'Operators which have an $H^{\infty}$ functional calculus', in: Miniconference on Operator Theory and PDE, Proc. of the Center for Math. Analysis (Australian National University, Canberra, 1986) pp. 210-231.

[35] Y. Nakamura, 'Classes of operator monotone functions and stieltjes functions', in: Operator Theory: Advances and Applications 41 (Birkhauser, Basel, 1989) pp. 395-404.

[36] E. Nelson, 'A functional calculus using singular laplace integrals', Trans. Amer. Math. Soc. 88 (1959), 400-413.

[37] A. Pazy, Semigroups of linear operators and applications to partial differential equations (Springer, New York, 1983).

[38] R. S. Phillips, 'On the generation of semigroups of linear operators', Pac. J.Math. 2 (1952), 343-369.

[39] E. I. Pustylnik, 'On functions of a positive operator', Math. USSR Sbornik 47 (1984), $27-42$.

[40] M. Reed and B. Simon, Methods of modern mathematical physics II (Academic Press, New York, 1975).

[41] W. Ricker, 'Spectral properties of the Laplace operator in $L^{p}(\mathbf{R})$ ', Osaka J. Math. 25 (1988), 399-410.

[42] _ 'An $L^{1}$-type functional calculus for the Laplace operator in $L^{p}(\mathbf{R})$ ', J. Operator Theory 21 (1989), 41-67.

[43] M. Rosenblum and J. Rovnyak, Hardy classes and operator theory (Oxford University Press, Oxford, 1985).

[44] H. Tanabe, Equations of evolution (Pitman, London, 1979).

[45] J. Watanabe, 'On some properties of fractional powers of linear operators', Proc. Japan 
Acad. 37 (1961), 273-275.

[46] D. V. Widder, The Laplace transform (Princeton University Press, Princeton, 1946).

[47] - Introduction to transform theory (Academic Press, New York and London, 1971).

[48] A. Yagi, 'Coincidence entre des espaces d'interpolation et des domaines de puissances fractionnaires d'operateurs', C. R. Acad. Sci. Paris Ser. I Math. 299 (1984), 173-176.

[49] K. Yosida, 'Fractional powers of infinitesimal generators and the analyticity of the semigroups generated by them', Proc. Japan Acad. Ser. A Math. Sci. 36 (1960), 86-89.

[50] - Functional Analysis (Springer, Berlin, 1978).

Matematisk Institut

Universitetsparken 5, DK-2100

Kobenhavn $\phi$

Demark

Ohio University

Athens, Ohio 45701

USA
Ohio Northern University Ada, Ohio 45810

USA 